\title{
FoRire, folie, chaos, désastre : vers un nouvel espace comique dans le théâtre contemporain
}

\section{Mireille Losco-Lena}

\section{Q OpenEdition}

1 Journals

Édition électronique

URL : http://journals.openedition.org/recherchestravaux/290

DOI : 10.4000/recherchestravaux.290

ISSN : 1969-6434

Éditeur

UGA Éditions/Université Grenoble Alpes

\section{Édition imprimée}

Date de publication : 30 mai 2005

Pagination : 239-249

ISBN : 0151-1874

ISSN : 0151-1874

Référence électronique

Mireille Losco-Lena, «FoRire, folie, chaos, désastre : vers un nouvel espace comique dans le théâtre contemporain », Recherches \& Travaux [En ligne], 67 | 2005, mis en ligne le 30 septembre 2008,

consulté le 08 septembre 2020. URL : http://journals.openedition.org/recherchestravaux/290 ; DOI : https://doi.org/10.4000/recherchestravaux.290 
Mireille LOSCO-LENA

Université Stendhal - Grenoble 3

\section{FoRire, folie, chaos, désastre: vers un nouvel espace comique dans le théâtre contemporain}

Un certain nombre d'écritures dramatiques entretiennent aujourd'hui une relation privilégiée avec un comique de contraste aussi surprenant que déroutant, puisqu'il se met au service d'une représentation douloureuse voire franchement désastreuse du monde. Suivant l'intuition beckettienne selon laquelle "rien n'est plus drôle que le malheur ${ }^{\mathrm{I}}$ ", ce théâtre se construit sur une profonde perturbation épistémologique dont il faut sans doute trouver les prémices chez les romantiques. Le "comique authentique», écrivait en effet I836 le philosophe Friedrich Theodor Vischer, «s'attaque à la vraie grandeur ${ }^{2}$ ». Contredisant à la fois l'héritage d'Aristote et l'esthétique de Hegel, Vischer poursuivait: «La véritable plaisanterie ne doit jamais s'en prendre à un sublime purement apparent mais toujours à un sublime véritable.» Délogeant le comique de la sphère du divertissement mais aussi d'une forme de gaieté légère, la pensée moderne du rire se voue au paradoxe et à un certain désarroi esthétique et éthique. Mais un tel désarroi, parfois désigné par le terme d' "humour", s'avère moins un frein qu'un nouveau moteur dans la représentation; il engage un renouvellement profond de la poétique des genres et une redistribution des qualités mimétiques et éthiques des modes fondamentaux de la représentation. Dès la fin du XIXe siècle avec l'Ubu Roi de Jarry, puis à la fin des années 1920 avec Victor ou les enfants au pouvoir de Vitrac, le théâtre s'essaie avec succès - et scandale - au jeu d'une coïncidence anti-aristotélicienne entre le comique et la douleur. En conjuguant l'esprit farcesque avec la représentation de la violence politique ou domestique, les

I. S. Beckett, Fin de partie, Paris, Minuit, 1957, p. 33.

2. Fr. Th. Vischer, Le Sublime et le Comique, projet d'une esthétique [1836], traduction et présentation de M. Espagne, Paris, Kimé, 2002, p. I30.

3. Ibid., p. 132 . 
dramaturges font définitivement péricliter les catégories génériques en s’appropriant pleinement l'esprit du grotesque issu des romantiques, ce comique de l'exagération et de la laideur qui fait grimacer l'homme. Certes, ces deux pièces restent des exceptions en leur temps, et il aura fallu un siècle de grandes désillusions pour que la pratique d'un théâtre comique de l'horreur et de la douleur se généralise, et sans doute même se radicalise. Le comique se découvre en effet une fonction nouvelle, celle de porter la représentation du monde à son plus haut point de chaos et de défaite du sens. Loin de correspondre à une mise en représentation euphorique et édulcorée du réel, il est un geste négatif qui entraîne tout avec lui et ne laisse souvent aucune planche de secours: le monde y est montré comme un carnaval qui est en passe de perdre tout caractère festif. Si l'on en croit la Pochade millénariste d'Eugène Durif4, carnaval et apocalypse vont jusqu'à se recouvrir l'un l'autre. Ce comique entretient alors un rapport privilégié avec le tragique, dans la mesure où il ne s'inscrit dans aucune "perspective explicative, finaliste et compensatrices».

\section{Pertinence du comique}

La représentation comique contemporaine, malgré sa part de fantaisie et de distorsion du réel, se voit hissée au rang de mimésis exemplaire du chaos du monde. "Malgré» ou plutôt " grâce à » : dans la logique mimétique de ces dramaturgies, la fantaisie théâtrale produit une folie qui s'érige insolemment en analogon de la folie de l'Histoire. Le dramaturge autrichien Peter Turrini décrit ainsi ce processus:

Je ne crois plus qu’au jeu, à la fiction. [...] Le théâtre n'est que faux-semblant. En avouant ce faux-semblant, des sensations vraies redeviennent possibles. [...] J'ai la nostalgie d'un théâtre qui n’ait rien à faire avec la réalité [...] et qui pourtant ouvrirait le regard sur elle. ${ }^{6}$

Se permettre au théâtre tous les excès, toutes les outrances, c'est pour Turrini commencer à saisir la vérité d'une Histoire catastrophique dont la machine s'est complètement emballée. On pourrait croire ici à un simple retour à l'usage classique de la comédie, qui dénonce et critique les travers des hommes. Ce serait manquer la spécificité de ces pièces dans lesquelles l'excès comique n'est plus un procédé de grossissement des travers humains permettant au spectateur de les déceler et de les sanctionner par le rire. La fantaisie se fait au contraire la juste métaphore d'un monde ou d'une humanité eux-

4. E. Durif, Pochade millénariste, Arles, Actes Sud-Papiers, 2000.

5. C. Rosset, "Le rire exterminateur (Esthétique du pire II)», Logique du pire, éléments pour une philosophie tragique (197I), Paris, Presses universitaires de France (Quadrige), I993, p. I73.

6. P. Turrini, préface à La Bataille de Vienne, texte français de H. Christophe, Arles, Actes Sud-Papiers, I995, p. 9. 
mêmes dénaturés, quoiqu'ils continuent à se cacher derrière des masques respectables - «cette bonhomie doucereuse qu'on attribuait toujours à l'Autriche 7». Dans son monologue Enfin la fin, par exemple, Turrini s'empare du registre carnavalesque pour renvoyer au monde contemporain une image à la fois folle et vraie de son inanité. Un homme se présente à nous en scène un pistolet à la main, et annonce simplement: «Je vais compter jusqu’à mille et me tuer ${ }^{8}$.» Au cours du décompte qu'il va effectuer jusqu'au geste final, il livre au public des fragments de récits de vie tous animés par un mouvement frénétique qui fait irrésistiblement surgir le rire; sa vie apparait comme un catalogue d'incongruités, constitué tout à la fois d'accumulations et de contradictions:

À dix-sept ans j’ai caressé le rêve de devenir champion du monde de vol à ski, et abandonné ce projet, qui m’aurait empêché d'être élu pape. [...] J'ai conçu des hold-up de banque, je voulais danser sous les palmiers avec les filles des mers du Sud et envisageais aussitôt la vie monacale.9

Le comique naît autant de la fantaisie du propos, de son irréalisme, que de la rhétorique proprement humoristique qui l'anime: la rhétorique de la contradiction ou du retournement inattendu débouche chaque fois sur une considération encore plus fantaisiste, encore plus folle. Chaque souvenir passé en revue est ainsi frappé par un mouvement de surenchère contrariante, jusqu'à ce que de la confession touche au non-sens, à l'indifférenciation du chaos et à la perte de toutes les valeurs: «Dans un passage de la Bible s'immisçaient les blagues dont j'avais égayé toute une table ${ }^{\mathrm{IO}}$. " Le «faux-semblant» du comique s'érige en instrument de dévoilement du réel, et la fatrasie devient l'analogon d'une représentation stupéfiante du monde contemporain comme monceau de déchets, immense décharge publique:

D'ici peu, le monde débordera de cadavres, de réfrigérateurs et autres appareils électriques non éliminables. Une montagne de cadavres, de réfrigérateurs, de grille-pain, de magnétoscopes. Et au sommet de cette montagne, je suis assis, moi [...]. Un grille-pain et une antenne de télé s'enfoncent dans mon cul éternellement vivant et me condamnent à l'immortalité. II

La fantaisie de la parole comique est à l'image d'une société de consommation dont l'homme est l'ultime déchet. La singularité de l'histrion en scène se fait alors exemplaire de l'humanité contemporaine. En chemin, Turrini aura

7. Ibid., p. 7 .

8. P. Turrini, Enfin la fin, texte français de H. Christophe, Arles, Actes Sud-Papiers, I998, p. 35 .
9. Ibid., p. 40.
Io. Ibid., p. 53.
II. Ibid., p. 47. 
obligé son public à réfléchir à son propre rire et à en peser la part de similitude avec la folie de son monde. Car au moment où le bouffon juge que le rire du public lui est indéfectiblement acquis, il lui renvoie une image extrêmement contrariante de la communauté des rieurs:

On m’invita à prononcer le discours anniversaire d'un industriel juif [...] J'ai poursuivi: il n'y a qu'une seule chose qui soit pire que les nazis de race pure: ce sont les juifs de race pure. Ils me dévisagèrent, effrayés, puis éclatèrent de rire. Ils rirent, rirent, rirent. Je me noyai dans cet océan de rires, et ris avec eux. ${ }^{\mathrm{I2}}$

Le rire est bien la marque de la déroute de la pensée, le signe d'une indifférenciation générale des valeurs qui viennent en dernier ressort s'échouer dans la vaste déchetterie du monde.

\section{La farce au-delà de la tragédie}

Bien sûr le délire de théâtre demeure un travail de fiction ludique. Mais ce type de fictionnement se révèle un mode mimétique beaucoup moins irréaliste que ce que l'usage de la tradition en avait fait. D'une certaine façon, la folie comique se voit prise au pied de la lettre. Un certain nombre de textes nous amènent à nous demander si ce théâtre ne prétend pas parler du monde à un endroit où les formes dites «sérieuses" risquent d'échouer: l'endroit de la déréliction absolue. Ce phénomène est manifeste dans la dramaturgie algérienne contemporaine, qui tente de rendre compte par la farce de la folie meurtrière qui a ravagé le pays depuis le début des années 1990. Dans sa pièce intitulée Yasmine ${ }^{13}$, par exemple, Omar Fetmouche présente une jeune fille enlevée de son village par un groupe d'islamistes, le jour de l'Aïd; après avoir été violée, elle retourne chez elle enceinte, mais personne ne veut plus d'elle: elle porte la malédiction. La jeune fille s'enfuit dans la montagne pour accoucher de son «monstre" sur le tombeau des ancêtres. Cette pièce, extrêmement fragmentaire, renoue avec la tragédie méditerranéenne, mais n’en restitue que des images désarticulées, comme inappréhendables par ce logos inventé par les Grecs. L’ancien chœur se voit réduit à deux citoyens-témoins qui finissent par reconnaître l'impossibilité d'achever la fable et surtout d'en tirer une morale tragique. Le récit final de la pièce nous entraîne dès lors dans une déroute violemment comique où l'absurde a pris le pas sur toute rationalité. Le chœur conte la folie qui s'est emparée du village, pris d'une peur aussi panique qu'aberrante de la contamination du mal. La chasse aux sorcières, commencée avec Yasmine, se propage jusqu’au délire, ouvrant la tragédie au grotesque:

I2. Ibid., p. 45 .

13. O. Fetmouche, Yasmine, manuscrit consultable au Centre de ressources de troisième bureau à Grenoble, p. Io. 
pour «arrêter la propagation du mal», «il faut raser le village ${ }^{\mathrm{I} 4}$ »; et comme cela ne suffit pas, «il faut déshabiller le peuple pour l'intérêt du village ${ }^{15}$ ». Cette pièce violente, véritable cauchemar, s'achève dans une atmosphère de délire comique avec une scène complètement ubuesque où la folie paranoïaque des villageois atteint des sommets de bouffonnerie. Cette bifurcation inattendue de la dramaturgie est extrêmement parlante: la tragédie ne peut finir qu'en comédie, et même en grosse farce, à partir du moment où l'horreur est devenue excessive. Comme si, dans l'excès d'horreur, la tragédie n'avait jamais réussi à aller aussi loin que le réel, et que sur la voie de l'excès, le comique offrait un mode de représentation beaucoup plus puissant. Le surgissement de la farce est alors le signe d'un passage à l'extrême, une façon de franchir le seuil de l'insensé.

Cette affinité entre le comique et l'inconcevable horreur place ce théâtre sur un terrain qu'Aristote avait banni de la tragédie: le miaron, que l'on traduit par le «répugnant» mais qui correspond aussi bien, selon Myriam Revault d'Allonnes, à l'«injustifiable ${ }^{16}$ ». Dans le chapitre $\mathrm{I} 3$ de La Poétique, Aristote associe en effet le miaron à une configuration de la fable qui montrerait «des justes passer du bonheur au malheur ${ }^{17}$ ». Des personnages qui, telle la Yasmine de Fetmouche, n'ont en aucun cas mérité l'avalanche insensée des douleurs ni l'expérience-limite de l'horrible, n'ont pas droit de cité dans la tragédie: cette dernière ne peut accueillir que des fables vraisemblables du point de vue de la logique et de l'éthique. Or le miaron confronte le spectateur à la défaite de la compréhension et à la perte du jugement:

Sous les espèces de l'inadmissible, miaron désignerait une forme particulière de l'invraisemblable, dont, à l'évidence (dèlon), la tragédie ne saurait s'accommoder. ${ }^{18}$

Non seulement le miaron empêche de faire accéder la douleur humaine à l'intelligibilité, mettant en crise la logique, mais encore il contrevient au sens de l'humain (philanthrôpia), et met par là en crise l'éthique: le principe de "juste rétribution" s'y voit fondamentalement bafouér ${ }^{9}$. Or que nous montre Omar Fetmouche, avec le basculement final de sa tragédie dans la farce? Qu'arrive un seuil dans l'horreur où le comique seul peut prendre en charge le miaron, c'est-à-dire la dimension de l'invraisemblable logique et éthique.

I4. Ibid., p. I4.

15. Ibid., p. I6.

I6. M. Revault d'Allonnes, «Peut-on élaborer le terrible?», Fragile humanité, Paris, Aubier (Alto), 2002, p. I84.

17. Aristote, La Poétique, édition de R. Dupont-Roc et J. Lallot, Paris, Seuil (Poétique), 1980, p. 77.

18. R. Dupont-Roc et J. Lallot, commentaire de La Poétique, ibid., p. $24 \mathrm{I}$.

19. Ibid., p. 242-243. 
Aussi déroutant pour la pensée cela soit-il, le comique devient l'outil le plus adéquat pour représenter l'excès d'horreur, pour une raison simple mais vertigineuse: ses propriétés fondamentales - bien connues - correspondent très exactement aux propriétés des événements terribles: l'insensé d'une part, et d'autre part la mise en déroute de l'éthique, la déliaison même de la philanthrôpia, dans la mesure où le comique exige distance et recul et qu'il refuse le principe d'identification, c'est-à-dire d'un sens de la communauté avec les personnages. Le fonctionnement du comique est exactement le même que celui du scandale de l'horreur, car se jouent dans les deux phénomènes à la fois une incapacité de comprendre et une perte de ce que Myriam Revault d'Allonnes nomme le sensus communis, "le partage du monde ${ }^{20}$ ». Du point de vue de la "technique» de la représentation - je ne parle pas encore de l'étrange aporie affective ou morale à laquelle cette considération va nous mener -, le comique serait le seul mode mimétique à même d'approcher l'insupportable passage des justes «du bonheur au malheur».

Cette découverte poétique, qui a cheminé en Europe depuis le romantisme, constitue une véritable rupture épistémologique. Elle semble toutefois infiniment plus naturelle aux Algériens, et en cela, leur pratique du comique peut éclairer la nôtre puisqu'elle en fait apparaitre les enjeux avec une clarté éblouissante. Ainsi, dans son unique pièce de théâtre, Normal!, le cinéaste Merzak Allouche fait un usage généreux du comique qui vient lui aussi occuper le terrain du miaron, celui de la barbarie de la fin du XX siècle en Algérie. La scène s'érige en microcosme désolé de la société contemporaine: c'est un espace-poubelle, peuplé de quatre personnages inscrits dans une temporalité schématique et raccourcie: une seule journée des personnages correspondra à la "décennie noire», de la fin des années I980 à l'émergence de l'islamisme et au début des massacres. La pièce s'achève dans un imbroglio de comédie qui semblerait bien éculé s'il ne coïncidait pas, et de façon saisissante, avec le réel. Dans le jeu des masques et des intrigues, la confusion entre le pouvoir étatique et les terroristes islamistes atteint son comble: «Nous ne savons plus qui est qui... Qui fait quoi... L'embrouille normale est totale, et vous... ${ }^{21} »$ Le jeu excessivement drôle du "qui est qui» ou quiproquo de comédie est un analogon quasi réaliste de la réalité excessivement horrible du «qui tue qui» de l'Histoire contemporaine algérienne. C'est donc tout naturellement que le comique de théâtre se voit questionné de l'intérieur de la pièce, un peu comme si les personnages n'en revenaient pas de se découvrir pris dans la

20. M. Revault d'Allonnes, op. cit., p. 197.

2I. M. Allouache, Normal!, manuscrit consultable au Centre de ressources de troisième bureau à Grenoble, p. 59 . 
fable d'Ubu Roi de Jarry: "Ça devient franchement ubuesque!» s'exclame un personnage. Ce à quoi un autre répond: «Nous sombrons dans la névrose $[\ldots]^{22}$.» La référence psychiatrique n'est pas sans importance. Elle éclaire le recours au comique dans son lien au miaron, c'est-à-dire dans sa capacité à saisir un réel qui n'est plus ni représentable ni même appréhendable par la raison et qui a des affinités avec la folie:

Tout le monde décapite, tout le monde mutile [...] Nous nous haïssons à la vie à la mort, enveloppés dans le linceul de cette damnation qui a fondu sur nous sans que l'on en sache les causes [...] Ce cataclysme qui a fait de nous des tueurs, des assassinés, des couards, des traîtres, des lâches [...]. ${ }^{23}$

La farce n'est plus une esthétique de l'exagération et de la caricature: elle est dictée directement par l'Histoire elle-même, dont les horreurs excèdent infiniment les excès formels du dramaturge - un peu comme si la fiction était prise de court par la folie réelle des hommes. Le comique surgit à l'endroit précis où la raison ne peut plus donner de "causes» à la catastrophe tragique, là où aucun argument ni aucun fait ne parviennent plus à expliquer l'ampleur du désastre. Dans cet échec avoué des liens de causalité - la fameuse exigence aristotélicienne de "vraisemblable»-, la représentation échappe à la tragédie et se place sur le terrain de la farce, où l'irrévérence radicale du comique fait jaillir une représentation du chaos. Encore une fois, le comique vient se placer très exactement dans le lieu laissé vacant par l'ancien système des genres hérité d'Aristote, le lieu de l'excès de l'horreur. Un excès non seulement des faits - la tragédie est parfaitement capable de représenter des faits d'une violence inouie - mais aussi de la défaite des liens de causalité et de la dissolution du muthos.

\section{Comique et crise de la représentation}

Il apparaît ainsi que l'épanouissement de ce type de comique répond à une crise de la représentation. Cette réflexion de Malek Alloula, le frère du grand dramaturge algérien Abdelkader Alloula assassiné en 1994, peut en faire apparaître les enjeux:

Car, comment évoquer cette situation-là [la décennie noire] sans qu'aussitôt les mots et les phrases employés ne soient renvoyés à une confondante irréalité qui ferait presque douter de notre qualité d'êtres pensants et parlants?24

22. Ibid., p. 52.

23. Ibid., p. 6I.

24. M. Alloula, "Avant-propos» à En mémoire du futur, Pour Abdelkader Alloula, Arles, Actes Sud (Sindbad), I997, p. I2. 
L'expérience subjective de l'horreur confronte les hommes à un violent sentiment d'irréalité, quelque chose de la sensation très intime de l'effondrement du monde, et dont les outils logiques de la représentation ne peuvent pas rendre compte. Il est ainsi remarquable qu'aujourd'hui les plus grandes réussites esthétiques en matière de théâtre comique de l'horreur se rencontrent tout particulièrement dans des pays ou chez des auteurs qui ont fait l'expérience intime et extrêmement meurtrissante de l'effondrement de leur monde: un effondrement objectif d'abord, souvent lié au massacre, au totalitarisme ou à de violentes crises politiques, mais aussi un effondrement subjectif qui correspond à une défaite intérieure des valeurs qui civilisent et humanisent tout un chacun. En Europe, les totalitarismes nazi et communiste ont ainsi alimenté des dramaturgies paradoxales du rire et de l'horreur. Dans Mein Kampf notamment, le dramaturge George Tabori fait du comique un puissant outil d'investigation du cauchemar nazi; par le recours à de multiples formes de comique héritées de la tradition (farcesque du "bas-corporel», humour juif mais aussi sketches dans l'esprit du cinéma burlesque de Mack Sennett), il s'inscrit en plein dans la crise de la représentation liée à la Shoah, que Myriam Revault d'Allonnes problématise ainsi :

Comment représenter une négation d'humanité? Comment mettre une inhumanité en rapport avec une humanité puisque la possibilité même de la "représentation » est donnée, classiquement, sous la condition d'une vraisemblance éthique instrinsèquement liée à la capacité imaginative de reconnaître en l'autre un semblable qui appartient lui aussi au genre humain?25

C'est justement cette impossible mise en rapport de l'humanité du juif Shlomo Herzl avec l'inhumanité de Hitler que raconte le Mein Kampf de Tabori, et c'est aussi de cette impossibilité que surgit le comique bouffon et aberrant de la pièce. Le non-sens hilarant de la pseudo-histoire d'amour fils /mère entre Hitler et Shlomo joue dans la pièce comme un révélateur esthétique de l'insensé de l'Histoire; il vient proposer en partage au spectateur cette expérience intime de la "confondante irréalité» dont parlait Malek Alloula. Si les événements terribles et injustifiables de l'Histoire mettent en crise le sensus communis tant ils paraissent inconcevables, le mode de représentation comique tente de reconstruire un espace commun, un lieu de partage entre scène et salle qui opère moins sur le terrain de la compréhension rationnelle des événements, définitivement échouée semble-t-il, que sur celui de la perception subjective de leur «irréelle réalité».

La fable théâtrale opère ainsi à un tout autre niveau que le discours de l'histoire ou de la philosophie; elle échappe au fonctionnement mimétique

25. M. Revault d'Allonnes, op. cit., p. I85. 
proposé par Aristote et emprunte les chemins de la parabole, ou, plus largement, de ce que Jean-Pierre Sarrazac appelle le «détour ${ }^{26}$ ». Un détour qui, précisons-le, vise moins l'analyse des faits - comme le voulait par exemple la parabole brechtienne - que la recréation d'un partage des émotions face à des événements inconcevables. Cette configuration peut avoir quelque chose d'absurde ou de provocateur dans la mesure où, bien évidemment, des événements comme les crimes du nazisme ou les massacres algériens n'ont en soi rien de comique, bien au contraire. C'est pourtant l'ultime pari paradoxal que font ici les auteurs dramatiques, et sans doute le plus périlleux de tous. Car si le détour comique a, comme nous avons essayé de le montrer, une pertinence du point de vue de la technique de la représentation, il suscite de toute évidence une grande perturbation épistémologique: il a toujours été globalement pensé à la fois dans un lien au plaisir voire à la gaieté, et dans une opposition au tragique et à la douleur. Comment dès lors concevoir qu' il puisse être l'agent d'une reconstruction du sensus communis autour de la représentation du désastre - d'un désastre non édulcoré?

C'est pourtant par un franc détour comique que, dans sa pièce intitulée La Cheminée, le Bulgare Margarit Minkov nous donne à partager l'expérience de l'enfermement communiste et la paranoïa d'individus broyés par le totalitarisme. Étonnamment drôle et angoissante tout à la fois, la pièce est écrite sous la forme d'une longue scène "de ménage» ininterrompue dont la portée parabolique est claire. Iris et Henri dialoguent, crient, se disputent, se terrent, mais leurs querelles intimes n'ont que peu à voir avec une scène conjugale ordinaire. La mésentente porte sur l'existence des objets et des êtres qui les entourent ou les ont entourés. Par exemple, ils ont eu un animal domestique qui s'est jeté par la fenêtre: était-ce vraiment un chien? et s'est-il suicidé? La ville de Chicago, où Iris rappelle qu'ils sont allés en voyage de noces, existe-telle? Iris téléphone à son amie Hilda: est-ce vraiment Hilda? Ne serait-ce pas plutôt Anna, comme cette dernière le prétend? Et pourquoi Hilda-Anna estelle persuadée que Henri s'appelle John? etc. Dans la représentation comique suscitée par cette inscription permanente de l'absurde, le réel qui nous est montré n'est plus une chose stable, certaine; c'est un espace d'interrogations et de désaccords sans fin, un monde qui ressemble à des sables mouvants dans lesquels les personnages s'enfoncent inéluctablement. Minkov montre l'impuissance radicale des individus face à des forces historiques et politiques qui ont ravagé les êtres dans le plus profond de leur psyché. La pièce ne s'attache pas plus que celle de Tabori à faire l'impossible récit d'une Histoire désastreuse; en opérant au niveau de l'intime, elle nous donne à partager le désastre

26. J.-P. Sarrazac, La Parabole ou l'enfance du théâtre, Paris, Circé (Penser le théâtre), 2002. 
psychique engendré par l'Histoire: la perte de la capacité à penser et à (se) représenter le monde. Tout ce qui peut être formulé par les personnages, c'est leur impossibilité de trouver des mots à la hauteur de la déflagration intime: "L'histoire ne peut pas être réduite à de simples mots $[\ldots]^{27}$." Il apparait dès lors que l'écriture comique, réponse à une crise de la représentation, s'approprie pleinement la problématique du sublime naguère attachée aux genres dits «sérieux». L'infinie irréalité qui s'attache à la représentation comique signale en effet à tout moment que le désastre déborde infiniment toutes ses représentations. En investissant le champ de l'«inimaginable» et de l'«indicible», le comique contemporain fait du sublime son horizon permanent. Il devient le lieu d'une expérience du vertige dont Baudelaire, il y a un siècle et demi, faisait déjà le récit médusé à propos d'une pantomime anglaise: "Il m’a semblé que le signe distinctif de ce genre de comique était la violence ${ }^{28}$. La caractéristique des personnages de la pantomime anglaise est en effet qu' «ils s'exercent aux grands désastres ${ }^{29}{ }^{»}$; dès lors le vertige fait partie intégrante de l'expérience comique: «c'était le vertige de l'hyperbole ${ }^{30}$ ». Et Baudelaire conclut: "Qu'est-ce que ce vertige? C'est le comique absolu ${ }^{31}$.»

\section{Quelques hypothèses pour finir...}

La configuration esthétique de cette représentation est extrêmement perturbante: le comique est parfaitement adéquat, du point de vue de la technique de la représentation, à dire le désastre, mais il est parfaitement contraire au sentiment douloureux que le désastre suscite en nous; pourtant il est à même d'offrir au spectateur un partage de la "confondante irréalité» du désastre, et par cette voie, de lui donner excès à la commotion du sublime - c'est-à-dire de récupérer peut-être, au bout du chemin, la fameuse philanthrôpia aristotélicienne. Serait-ce à dire que, face aux plus grands désastres, seul le comique peut parvenir à recréer un partage des émotions digne de ce nom, c'est-à-dire un partage qui ne sombre pas dans cette compassion confortable et douteuse que fustigeait déjà Rousseau en son temps? Cette proposition mériterait un examen prudent et beaucoup plus approfondi, et nous en resterons ici pour finir au seul stade des hypothèses. Â la fin de Mein Kampf, George Tabori invite le public à se questionner quant à une possible refondation du tragique

27. M. Minkov, La Cheminée, traduit du bulgare par T. Mileva, Paris, Les Solitaires intempestifs, 200 .

28. Ch. Baudelaire, «De l'essence du rire et généralement du comique dans les arts plastiques» (1855), Écrits sur l'art, Paris, Le Livre de Poche, I999, p. 297.

29. Ibid.

3o. Ibid., p. 298.

31. Ibid., p. 299. 
au sein même du comique. En effet, au moment où la farce rejoint le désastre réel de la Shoah, Lobkowitz vient conclure la pièce par la blague suivante: «Le premier larron gémit, suspendu sur la croix. Le deuxième larron lui demande: "Ça fait mal ?" Et le premier lui dit: "Seulement quand je ris" ${ }^{2}$ ". Par-delà son absurdité ou son non-sens apparents, qui conduisent au rire, cette blague ne laisse pas d'interroger et de perturber nos conceptions du comique et du tragique. Est-ce que vraiment seul le rire pourrait faire toucher aux plus violentes souffrances? Toute la théorie psychanalytique du comique, qui voit en lui un phénomène protecteur face à la douleur, se voit remise en question - et notre réflexion s'en trouve d'un coup bien désarmée. Il y a là une large matière à réflexion et à recherche, qui déborde largement l'analyse esthétique.

On émettra seulement ici une hypothèse qui reste à vérifier et à démontrer: le comique donnerait accès au miaron en évitant au spectateur de s'horrifier en chemin - trop tôt. Cela signifie qu'il ferait le pari du détour esthétique pour empêcher le spectateur de se détourner lui-même: tels les bouffons immémoriaux, il prendrait sur lui la part d'insensé, il endosserait la part de folie pour permettre au spectateur de parvenir au terme de la route. Et, scandale s'il en est, il ruserait, il nous ferait croire au caractère acceptable - et même risible! - de la représentation pour, au final, nous faire appréhender ce quelque chose de l'horreur qui échappe à toute représentation. Cette dimension de ruse ne veut pas dire qu'il s'agisse d'un faux comique, ni que nous ayions eu tort, rétrospectivement, d'avoir ri: peut-être faut-il au contraire avoir cédé au délire du rire pour que celui-ci nous mène à la pleine conscience du délire de mort. Peut-être faut-il faire confiance au diable du comique pour accéder à la vision diabolique. Car au bout du chemin, les pièces ne sauraient camouffler l'horreur du réel: là est l'exigence proprement éthique (ou philanthropique) de l'art - ce qui marque sa radicale différence avec la mauvaise blague qui nie le «terrible» et protège le rieur -, et peut-être le ferment d'un nouveau contrat tragique au sein de l'assemblée théâtrale. C'est là l'ultime hypothèse que nous formulerons: être parvenu au bout de la route encore vivant (c'est-à-dire, pour le spectateur, encore présent), c'est peut-être faire l'expérience d'une forme de catharsis. C'est avoir non seulement secoué l'indifférence à laquelle nous invitent les médias, mais aussi surmonté le refus auquel nous confronte le miaron, ainsi que la culpabilité dans laquelle le pathos nous enlise: en bref, c'est avoir renoué profondément avec le sentiment vivant du tragique.

32. G. Tabori, Mein Kampf (farce), texte français de A. Llamas, Arles, Actes Sud-Papiers, I993, p. 7I. 